\title{
5 Ethische Probleme in der Notaufnahme
}

\author{
Jan Padberg
}

\subsection{Ausgangslage}

Ethische Probleme und Herausforderungen begegnen uns täglich bei unserer Arbeit mit Notfallpatienten in Notaufnahmen. Diese meist kritischen Entscheidungssituationen sind keine seltenen Spezialfälle, die einen Spezialisten für Medizinethik erfordern, sondern werden von den gerade anwesenden Pflegekräften und Ärzten abgearbeitet. Typische Merkmale dieser ethischen Herausforderungen sind der hohe zeitliche Druck, Entscheidungen treffen zu müssen, und die häufig geringe Berufsund Lebenserfahrung der primär behandelnden Ärzte (Schöpke et al. 2014).

Die in diesem Zusammenhang getroffenen Entscheidungen haben häufig weitreichende Konsequenzen für die Patienten, es geht häufig tatsächlich um Leben oder Tod. In scharfem Kontrast zu der enormen patientenbezogenen Relevanz ethischer Probleme in der Notaufnahme stehen das geringe Problembewusstsein sowie fehlende ethische Kenntnisse und Fertigkeiten der Notfallmediziner. Auch sind die rechtlichen Rahmenbedingungen im Allgemeinen wenig bekannt. Anscheinend Ausdruck von Ignoranz oder Verdrängung dieser ethischen Herausforderungen sind die geringe individuelle Auseinandersetzung mit medizinethischen Themen und die defizitäre Aus- und Weiterbildung in diesem Gebiet. Gerade die praktische, lösungsorientierte Beschäftigung mit ethischen Problemen in der Notfallmedizin findet weder im Medizinstudium noch in der ärztlichen Weiterbildung ausreichend Berücksichtigung.

Die klinische Notfallmedizin in Deutschland hat sich in den letzten Jahrzehnten erheblich weiterentwickelt. Kontinuierlich wachsende Patientenzahlen und zuneh- 
mende Spezialisierung der klinischen Fachabteilungen haben zur Etablierung zentraler Notaufnahmen mit eigenen Leitungsstrukturen geführt. Die Herausforderung, gleichzeitig viele Patienten mit unterschiedlichsten Krankheitsursachen adäquat zu behandeln, mit der vollen Bandbreite von Befindlichkeitsstörungen bis hin zu akuter Lebensgefahr, hat die Professionalisierung der klinischen Notfallmedizin vorangetrieben. Dies zeigt sich unter anderem in der Cründung einer eigenen Fachgesellschaft, der Deutschen Gesellschaft für interdisziplinäre Notfall- und Akutmedizin (DGINA), in 2005 und der Einführung der Zusatzweiterbildung Klinische Notfall- und Akutmedizin der Ärztekammer Berlin nach dem EuSEM-Curriculum (European Society For Emergency Medicine) in 2014. Diese spezifische Professionalisierung der Notfallmedizin wird langfristig auch die medizinethische Kompetenz in deutschen Notaufnahmen verbessern.

\subsection{Probleme und Spannungsfelder}

\subsubsection{Der multimorbide, nicht-entscheidungsfähige Patient}

Fast täglich werden in größeren Notaufnahmen Patienten eingeliefert, bei denen die folgende ethische Problemkonstellation besteht:

1. kurzfristig lebensbedrohlicher Zustand

2. hohes Alter und/oder Multimorbidität und

3. unklarer mutmaßlicher Patientenwille.

Entscheidungsberechtigte und entscheidungsfähige Angehörige bzw. Betreuer sind häufig nicht anwesend oder erreichbar. In vielen Fällen haben sich weder der Patient noch die Angehörigen mit Wunsch und Willen des Kranken am potenziellen Lebensende beschäftigt bzw. es fehlte zuvor an einer praxisnahen Beratung durch fachkompetente Ärzte. Viele Ärzte haben außerdem nur begrenzte Kenntnisse über Möglichkeiten, Erfolgswahrscheinlichkeiten und Belastungen intensivmedizinischer Maßnahmen. Die Einlieferung solcher Patienten wird von vielen Ärzten und Pflegekräften der Notaufnahmen als nicht mehr angemessen oder sinnlos eingeschätzt. Hierbei werden allerdings die palliativmedizinischen und ethischen Herausforderungen und Nöte dieser Patienten nicht wahrgenommen oder ignoriert.

\subsubsection{Patientenverfügungen}

Bei vielen Notfallpatienten liegen keine Patientenverfügungen vor und, falls doch, entsprechen diese meist unterschriebenen Standardformulierungen (Sommer et al. 2012; Padberg et al. 2014).

Die Verfügungen sind häufig situativ nur an „den unmittelbaren Sterbeprozess“, „das Endstadium einer unheilbaren, tödlich verlaufenden Krankheit“ oder „die aller Wahrscheinlichkeit nach unwiederbringliche erloschene Einsichts- und Entscheidungsfähigkeit“ gebunden. Diese Bedingungen treffen aber in der Notaufnahme selten eindeutig zu. Häufiger hat der Patient lediglich eine kurz- bis mittelfristig schlechte Prognose und kann sich aufgrund der akuten Verschlechterung seiner kognitiven Fähigkeiten nicht an einer Therapieentscheidung beteiligen. 


\subsubsection{Betreuung}

Die Zahl der amtlich betreuten Personen ist in Deutschland in den letzten Jahren auf ca. 1,3 Millionen gestiegen, davon werden ca. 30-40\% durch Berufsbetreuer versorgt (Deinert 2013). Diesen Berufsbetreuern fehlen sehr häufig valide Informationen über den mutmaßlichen Patientenwillen, auf gezielte Nachfragen folgen meist indifferente Antworten wie z.B. „Machen Sie, was ärztlich sinnvoll ist“, die bei den akuten ethischen Entscheidungen nicht hilfreich sind (Padberg et al. 2014). Es fehlt bei vielen Betreuern die Bereitschaft, Verantwortung zu übernehmen.

\subsubsection{Verantwortung}

Ethische Entscheidungen in der Notfallmedizin fallen immer individuell für jeden einzelnen Patienten und sind daher nicht standardisierbar. Bei Ärzten, Pflegekräften und Rettungsdienstmitarbeitern existiert häufig eine relevante Unsicherheit in ethischen Entscheidungssituationen. Daher fällt es den Akteuren nicht selten schwer, Verantwortung zu übernehmen und diese wird, meist unbewusst, der weiterbehandelnden Einheit übergeben, um der unangenehmen Entscheidungssituation zu entkommen. Die Entscheidungsverantwortung wird wie eine „heiße Kartoffel“ möglichst rasch und vollständig weitergegeben. Am Ende dieser Kette stehen dann die Ärzte und Pflegekräfte der Intensivstationen, die sich gelegentlich fühlen wie auf einer „Station für ethische Akutfragen am Lebensende“. Zusammenfassend ist es ethische Aufgabe jedes beteiligten Handelnden, im Laufe des Behandlungs- und Entscheidungsprozesses zumindest einen Teil der Verantwortung zu übernehmen.

Insbesondere Ärzte müssen versuchen, alle von ihren Teammitgliedern gewonnenen Informationen zu erhalten, um dann ihre Einschätzung und geplante Entscheidung im Team zu besprechen und die Verantwortung zu übernehmen. Unter Umständen bleibt für diesen Prozess nur sehr wenig Zeit. Keinesfalls darf die Verantwortung, erneut wie die „heiße Kartoffel“, einfach an Angehörige des Patienten übertragen werden nach dem Motto „Sie müssen jetzt entscheiden, was wir noch machen sollen!“. Generell kann ein Spannungsfeld entstehen zwischen dem legitimen Ziel, einen Konsens im Cruppenprozess zu erzielen, und der bewussten Verantwortungsübernahme des Arztes.

\subsubsection{Zeit und Ressourcen}

Ethische Entscheidungen in der Notaufnahme haben aufgrund ihrer oben gezeigten Relevanz eine hohe Priorität und benötigen ausreichende zeitliche und personelle Ressourcen. In den immer häufigeren Überlastungssituationen konkurriert dieser Bedarf jedoch mit den Bedürfnissen anderer Patienten. Hier wird das ethische Prinzip der Gerechtigkeit berührt. Die Pflegekräfte und Ärzte in Notaufnahmen können nicht anders, als ihre Zeit und Konzentration auf die Patienten zu verteilen. Der Zeitrahmen wird also nicht nur durch die Krankheitsschwere und den Verlauf, sondern auch durch die Arbeitsbelastung bestimmt. Chronische Überlastung, Kommunikationsprobleme und mangelnde Wertschätzung der Mitarbeiter können nach der Bedürfnispyramide von Maslow (s. Kap. 23 „Erst kommt das Fressen, dann die Moral“) gute ethische Entscheidungen in kritischen Notfallsituationen dauerhaft verhindern. 
Im individuellen Fall geben vor allem die Schwere und Dynamik der Erkrankung den Zeitrahmen vor. Bei akuter Lebensgefahr und dem Ausfall von Vitalfunktionen können nur noch Sekunden für eine Entscheidung verbleiben. Üblicherweise werden parallel therapeutische und diagnostische Maßnahmen sowie eine ethische Einschätzung der Gesamtsituation erbracht. Nach anfänglicher Stabilisierung oder bei weniger zeitkritischen Situationen kann sich ein Zeitfenster öffnen, welches eine differenzierte Entscheidung ermöglicht. Die Zeit fungiert folglich als wichtige Determinante und Leitschnur ethischer Entscheidung in der Notfallmedizin.

Der Entscheidungsprozess wird also unter Umständen dem Zeitrahmen angepasst; eventuell ist es notwendig, die Entscheidung in mehrere Schritte aufzuteilen. Manchmal kann in der Notaufnahme der Entscheidungsprozess nur begonnen oder fortgeführt und nicht komplett abgeschlossen werden. Wichtig ist hier die offene Kommunikation mit den weiterbehandelnden Einheiten.

\subsubsection{Reanimationen im Krankenhaus}

In allen modernen Akutkrankenhäusern sind Reanimationsdienste mit Ärzten und Pflegekräften etabliert, die nach Alarmierung sofort zu den betroffenen Patienten eilen. Teilweise werden diese Dienste bei akut lebensbedrohlich erkrankten Patienten als Medical Emergency Teams genutzt.

Nicht selten ist der Patient wie erwähnt chronisch multimorbide erkrankt und hat eine erheblich eingeschränkte Lebenserwartung (Trzeczak 2013). Im arbeitsverdichteten Ablauf der bisherigen stationären Behandlung wird oft weder mit dem Patienten noch mit den Angehörigen über seinen Wunsch und Willen im Falle eines Atemoder Kreislaufstillstands gesprochen. Manchmal wird im Rahmen dieser Unsicherheit auch keine Herzdruckmassage vom Stationspersonal begonnen. Hier muss nun das Reanimationsteam sofort entscheiden und wird im Allgemeinen mit der Wiederbelebung beginnen in dem Wissen, dass dieses mit einer gewissen Wahrscheinlichkeit nicht dem mutmaßlichem Willen des Patienten entspricht. Hier kollidiert der absolute Zeitdruck mit dem unterstellten mutmaßlichen Willen des Patienten und einer möglichen Aussichtslosigkeit (futility) der Reanimationsbemühungen. Unter diesem hohen zeitlichen Druck und moralisch-ethischem Zwiespalt werden bisweilen bewusst mangelhafte Reanimationsmaßnahmen begonnen, sogenannte Scheinreanimationen, welche auch krankenhausintern als „slow-code“ oder „Anreanimieren“ bezeichnet werden. Diese Scheinreanimationen sind mit ethischen Prinzipien nicht zu vereinbaren.

\subsubsection{Der alkoholisierte Patient}

Die Alkoholintoxikation ist der häufigste psychiatrische Diagnosekomplex in deutschen Notaufnahmen (Kropp et al. 2007). Hier lohnt es, zwischen jungen, akut intoxikierten und chronisch alkoholkranken Patienten zu unterscheiden. Bei der Behandlung von alkoholisierten Jugendlichen und jungen Erwachsenen im Rahmen von exzessivem Rauschtrinken (binge drinking) steht die medizinische Therapie im Vordergrund. Bei diesen Patienten fehlt der intensive Daueralkoholkonsum, es gibt meistens familiäre Bezugspersonen und häufig im Verlauf auch Krankheitseinsicht und Kooperativität. 
Problematischer stellt sich die Alkoholintoxikation bei chronisch alkoholkranken Patienten dar. Die Patienten sind durch sehr hohe Alkoholspiegel, relativ zum Alkoholspiegel geringe Bewusstseinstrübung, mangelnde Kooperativität und Krankheitseinsicht gekennzeichnet. Nicht selten bestehen auch Obdachlosigkeit und ein desolater Pflegezustand. Die ethische Herausforderung manifestiert sich, wenn der Patient, alkoholisiert, aber gesprächs- und gehfähig, die weitere Behandlung abbrechen, also gehen will. In deutlichem Gegensatz stehen dann die ethischen Prinzipien der Autonomie (Patient will gehen), der Fürsorge (Abwendung gesundheitlicher Gefahren) und des Nichtschadens (Gefährdung durch Fixierung oder Sedierung).

\subsection{Fallbeispiel}

Eine 85-jährige Patientin wird von Altenpflegern eines Pflegeheims und mit dem Eintrag „AZVerschlechterung "im Pflegeüberleitungsbogen in die Notaufnahme überstellt. Hinweise auf eine Patientenverfügung, Vorsorgevollmacht oder amtliche Betreuung fehlen. Die Patienten ist kachektisch und somnolent (GCS 9) mit kritischen Vitalparametern (RR 70/30 mmHg, Puls 90/min, Atemfrequenz 14/min, Sauerstoffsättigung 82\%) und pulmonalen Rasselgeräuschen beidseits. Unter kontinuierlichem Monitoring erfolgt die intravenöse Gabe von Elektrolytlösungen. Auch mithilfe der sofort verfügbaren Blutgasanalyse bietet sich das klinische Bild eines septischen Schocks mit ausgeprägter Laktatazidose. Nach kurzer Besprechung mit der Krankenschwester und dem Assistenzarzt ruft der Oberarzt der Notaufnahme in dem Pflegeheim an. Dort erfährt er, dass der aktuell telefonisch nicht erreichbare Sohn eine Vorsorgevollmacht habe, jedoch sei der Ehemann der Patientin anwesend. In dem ca. zehnminütigen Telefonat bespricht der Oberarzt mit dem Ehemann die aktuell lebensbedrohliche Erkrankung und die therapeutischen Optionen in einem vereinfachten Stufenmodell. Der mutmaßliche Wille der Patientin wird unter anderem anhand der Frage „Was würde Ihrer Frau in dieser oder einer vergleichbaren Situation entscheiden?" versucht zu ermitteln. In dem Gespräch wird deutlich, dass eine Aufnahme auf die Intensivstation und organersetzende Maßnahmen, wie auch eine mögliche Reanimation, definitiv nicht von der Patientin gewünscht worden wären. Diese Einschätzung wird auch von der zuständigen Altenpflegerin geteilt. So entscheidet sich der Oberarzt nach kurzer Rekapitulation mit seinem Behandlungsteam zur Aufnahme der Patientin auf eine periphere Station, aber auch zur sofortigen Einleitung einer antibiotischen Therapie. Das Vorgehen wird im Notaufnahmebogen dokumentiert und mit dem weiterbehandelnden Stationsarzt besprochen. In den nächsten Behandlungstagen stabilisiert sich die Patientin kurzfristig. Die Therapiebegrenzung und Therapiezieländerung wird auf der Station mit dem Sohn und dem Ehemann der Patientin überprüft und bestätigt. Nach drei Tagen kommt es zu einem massiven peranalen Blutabgang und einer ausgeprägten Anämie. Nach erneuter Besprechung mit den Angehörigen erfolgt eine rein palliative Therapie unter Verzicht auf Transfusionen. Die Patientin stirbt noch am gleichen Tag. 


\section{4 lösungsansätze, Hilfsmittel und Strukturmodelle}

\subsubsection{Hilfsmittel und hilfreiche Faktoren}

Die etablierten Hilfsmittel bei ethischen Herausforderungen wie Ethikkonsile, Palliativkonsile, ethische Fallbesprechungen und Ethikkomitees sind in Notaufnahmen vor allem wegen des hohen Zeitdrucks zur Lösung akuter Probleme nur eingeschränkt geeignet (Padberg 2014). Die Entscheidungsmuster und das Problembewusstsein der Akteure sind sehr individuell, variabel und von persönlichen Erfahrungen geprägt, insbesondere im Vergleich zu standardisierten Behandlungspfaden (SOPs, Leitlinien) bei definierten Krankheiten. Dennoch lassen sich mehrere Faktoren nennen, die gute ethische Entscheidungen in der Notaufnahme fördern und erleichtern können: Es sind die Basisfaktoren, die auch eine hochwertige medizinische Behandlung ermöglichen. Das sind zunächst ausreichende personelle Ressourcen und eine adäquate räumliche Ausstattung. Es folgen offene, wertschätzende Kommunikation, positiver Teamgeist und eine entwickelte Fehlerkultur. Vorteilhaft in der Notaufnahme sind eine eigene ärztliche und pflegerische Leitung und ein definiertes ärztliches Team, welches aus festen Stellenanteilen und Rotationsstellen bestehen kann. All das ermöglicht und fördert die Teambildung, vermehrtes Engagement und die Identifikation mit der Notaufnahme.

Eine Einstufung oder Kategorisierung medizinischer Maßnahmen ist in der Kommunikation mit Patienten und Angehörigen hilfreich, da die Betroffenen meist nur geringe Kenntnisse über die medizinischen Optionen im Krankenhaus haben. So eröffnen formulierte Bilder wie „Rote Linien“ oder „Stufen einer Pyramide“ das Verständnis für Grenzen medizinischer Maßnahmen und erleichtern die Bestimmung des Patientenwillens (s. Abb. 3).

Re-

animation

Organersetzende

Verfahren wie

Dialyse, Beatmung

Intensivmedizinische Behandlung

(optional: Katecholamine,

Defibrillation,

non-invasive Beatmung)

Krankenhausstationäre Therapie

(optional: Operationen, Infusionen,

parenterale Ernährung, Chemotherapie)

Ausschließlich ambulante, ggf. palliative Therapie

(optional: Antibiotika,

enterale Ernährung, Chemotherapie)

Abb. 3 Stufenmodell medizinischer Maßnahmen 
In der Klinik sollte versucht werden, vorhandene ethische Kompetenzen zu nutzen. So können ethisch problematische Fälle aus der Notaufnahme mithilfe eines lokalen Ethikkomitees retrospektiv analysiert und besprochen werden. Dieses Vorgehen ermöglicht zwar keine Lösung akuter ethischer Probleme, kann aber eine systematische und vertiefte Analyse erleichtern, Lösungsansätze generieren und das Team der Notaufnahme in ihrem Lernprozess nachhaltig unterstützen.

In einer vergleichbaren Weise können auch Notfallseelsorger interne Fallanalysen unterstützen und so einen Beitrag zur Entwicklung fundierter und reflektierter Wertvorstellungen der Notaufnahmebelegschaft leisten.

Besonders in der Folge von Reanimationen sollte eine zeitnahe Nachbesprechung (Debriefing) medizinischer und medizinethischer Aspekte etabliert werden. Auch hier können angepasste Debriefing-Checklisten verhindern, dass ethische Gesichtspunkte unausgesprochen bleiben. Mortalitäts- oder M\&M-Konferenzen bieten eine weitere geeignete Plattform, um ethische Herausforderungen in der Notfallmedizin anhand eines konkreten Falls transparent für alle Krankenhausmitarbeiter zu analysieren.

\subsubsection{Checkliste Notfallpatienten}

Die aus Pflegekräften, Ärzten, Rettungsassistenten und Medizinethikern bestehende Arbeitsgruppe „Ethik in der Notfall- und Akutmedizin“ der DGINA hat 2014 eine „Checkliste Notfallpatienten“ als Hilfestellung zur Bearbeitung akuter ethischer Probleme entwickelt. Ausgangsüberlegung war die Frage, was ein berufserfahrener Ausbilder einem Anfänger an praktischen Ratschlägen und Know-how für die ersten Dienste im Rettungsdienst oder in der Notaufnahme mit auf den Weg geben könnte. Die Checkliste wurde im Kitteltaschenformat gedruckt, ist aber auch auf üblichen Smartphones lesbar und kann gut in Einarbeitungs- und Ausbildungskonzepten für die Notaufnahme oder den Rettungsmedizin eingesetzt werden. Die Datei steht kostenfrei unter www.dgina.de als PDF zum Download zur Verfügung.

\section{Checkliste Notfallpatienten der AG Ethik der DGINA}

Daten und Unterlagen

- Kontaktmöglichkeiten? (z.B. Angehörige, Hausarzt) Telefonnummer notieren, ggf. Angehörige zum Patienten (z.B. in die Notaufnahme) bestellen.

- Patientenverfügung, Vorsorgevollmacht, Betreuung, Palliativbogen? Dokumente beim Patienten sichern.

- Krankengeschichte, Vorbefunde, Pflegeunterlagen, Medikation? Dokumente beim Patienten sichern.

Behandlung: Indikation und Einwilligung

- Aussichtslosigkeit medizinischer Maßnahmen? Eine Erfolgswahrscheinlichkeit unter 1\% kann als Aussichtslosigkeit („futility“) gewertet werden.

- Mutmaßlicher Wunsch und Wille des Patienten? Aussagen von Bezugspersonen erheben und dokumentieren (z.B. wie hätte der Patient sich in einer mit der aktuellen vergleichbaren Situation entschieden?). 
- Konsens im Behandlungsteam und Konsens mit Angehörigen? Einschätzungen aktiv erfragen und - falls möglich - weitere Kompetenz hinzuziehen (andere Ärzte, Oberarzthintergrunddienste), aber Verantwortung übernehmen.

- Verantwortung für ethische Entscheidungen übernommen? Die Verantwortung für ärztliche Handlungsentscheidungen, z.B. zur Durchführung von oder Verzicht auf medizinische Maßnahmen, verbleibt beim behandelnden Arzt. Diese Verantwortung kann nicht auf Angehörige oder Amtsbetreuer übertragen werden.

\subsubsection{Kette guter ethischer Entscheidungen analog der „Chain of Survival“}

Ethische Entscheidungen in der Notfallmedizin treten nicht nur in der Notaufnahme auf und werden auch nicht nur dort entschieden. Wenn wir diese Entscheidungen beispielhaft bei einem nicht-entscheidungsfähigen Patienten betrachten, der aus einem Pflegewohnheim mit dem Rettungswagen und Notarzt in die Notaufnahme transportiert wird und nach Behandlung auf die Intensivstation kommt, so können wir eine Reihe von Akteuren identifizieren. Diese Reihe lässt sich mit der bekannten Überlebenskette „Chain of Survival“ (Cummins et al. 1991) vergleichen - auch die Kette guter ethischer Entscheidungen ist nur so stark wie ihr schwächstes Glied.

Die folgende Aufzählung skizziert die Reihenfolge der Akteure in einer Kette guter ethischer Entscheidungen:

1. Angehörige, Pflegedienst und/oder Betreuer

2. Rettungsassistenten und Notärzte

3. Notaufnahme

4. Normal- und Intensivstationen (s. Abb. 4).

Im Rahmen der jeweiligen ethischen Problematik sammelt jedes Teil dieser Kette relevante Informationen und wirkt am Entscheidungsprozess mit.

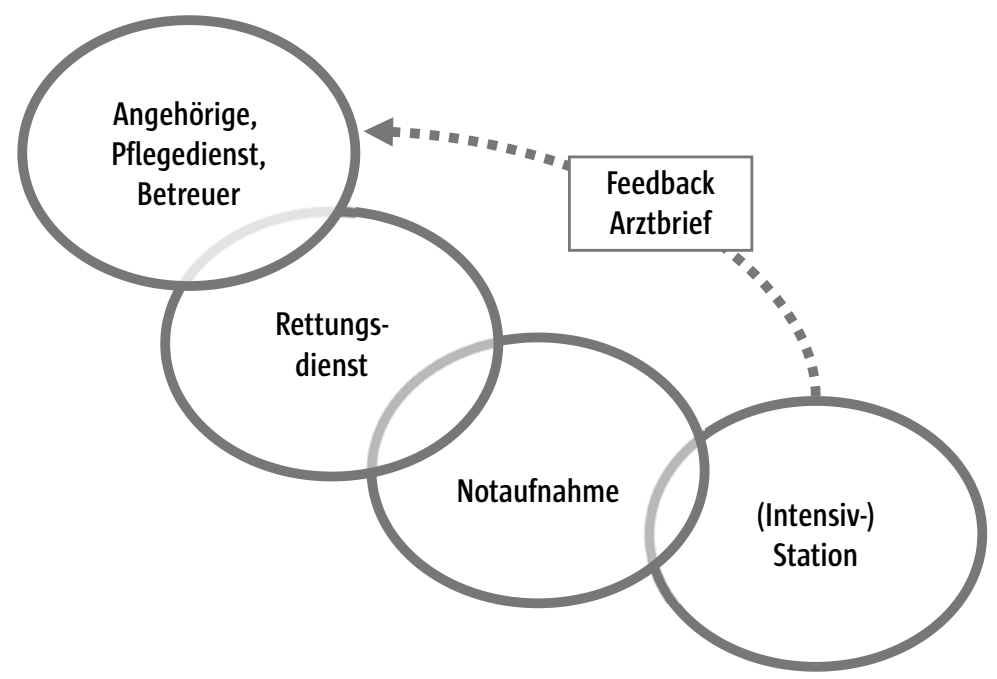

Abb. 4 Kette der Akteure ethischer Entscheidungen bei nicht entscheidungsfähigen Patienten 
In diesem Entscheidungsprozess spielt Kommunikation eine Schlüsselrolle, sowohl zwischen den oben genannten Akteuren als auch innerhalb des Behandlungsteams und immer wieder mit dem Patienten, wobei non-verbale Kommunikation (Patient verweigert Nahrungsaufnahme, wehrt ab, etc.) relevant werden kann.

Ferner sollten die im Behandlungsprozess gewonnenen Informationen über ethische Fragestellungen bei Entlassung aus dem Krankenhaus den Weiterbehandelnden z.B. im Arztbrief mitgeteilt werden, um einen weiteren, qualitativ hochwertigen ethischen Entscheidungsprozess zu unterstützen. Der meist gute Kontakt mit vor- und nachbehandelnden Einheiten, wie Rettungsdienst und Intensivstation, sollte zur gemeinsamen Betrachtung und Aufschlüsselung von kritischen ethischen Entscheidungssituationen genutzt werden.

\subsection{Aus- und Weiterbildung}

Die strukturierten Aus- und Weiterbildungsoptionen für Notaufnahmepersonal sind im deutschsprachigen Raum im internationalen Vergleich deutlich eingeschränkt. Exemplarisch sei nur das Fehlen eines Facharztes für Notfallmedizin in Deutschland erwähnt. Spezielle Curricula zu Ethik in der Notfallmedizin fehlen. Dennoch existieren verschiedene Optionen, die Qualität ethischer Entscheidungen in der Notfallmedizin zu fördern.

Die Fachweiterbildung für Pflegekräfte z.B. „Dipl. Experte/in Notfallpflege NDS HF“ in der Schweiz oder die berufsbegleitende zweijährige Fachweiterbildung Notfallpflege nach den Empfehlungen der DGINA umfassen auch notfallrelevante Themen der Medizinethik.

Medizinethik ist im Studium der Medizin als prüfungsrelevantes Fach fest etabliert. Problematisch ist die fehlende praktische Erfahrung der Studenten zum Kurszeitpunkt und damit verbunden der schwierige Transfer von Kenntnissen und Fähigkeiten Jahre später in die eigene Berufspraxis.

Eine sinnvolle Option ist sicher die berufsbegleitende Ausbildung einzelner Teammitglieder der Notaufnahme zum Ethikberater/in im Gesundheitswesen nach dem Curriculum „Ethikberatung im Krankenhaus“(Simon et al. 2005; http://www.ethikkomitee.de/zertifizierung/index.html).

\subsection{Schlussbemerkung}

Ethische Herausforderungen und Probleme sind alltäglich in Notaufnahmen relevant und somit ein elementarer Bestandteil der Notfallmedizin. Besprechen Sie die ethischen Probleme mit Ihren Patienten und deren Angehörigen und binden Sie Ihr Team sowie die vor- und nachbehandelnden Kollegen aktiv mit ein.

\section{Literatur}

Cummins RO, Ornato JP, Thies WH, Pepe PE (1991) Improving survival from sudden cardiac arrest: the "chain of survival" concept. Circulation 83(5): 1832-1847

Deinert H (2013) Betreuungszahlen 2012. BtPrax (6): 242-244 
Kropp S, Andreis C, te Wildt B, Sieberer M, Ziegenbein M, Huber T) (2007) Charakteristik psychiatrischer Patienten in der Notaufnahme. Psychiatr Prax 34: 72-75

Padberg I, Esser A, Lomberg L, Trzeczak S (2014) Ethische Probleme im Umgang mit Reanimation und Patientenverfügung in der Notaufnahme. Notfall Rettungsmed 6: 500-506

Schöpke T, Dodt C, Brachmann, M, Schnieder W, Petersen P-F, Böer I (2014) Statusbericht aus deutschen Notaufnahmen - Ergebnisse der DGINA Mitgliederbefragung 2013. Notfall Rettungsmed 17: 660-670

Simon A, May AT, Neitzke G (2005) Curriculum „Ethikberatung im Krankenhaus“. Ethik Med 17(4): 322-326

Sommer S, Marckmann G, Pentzek M, Wegscheider K, Abholz H-H, in der Schmitten I (2012) Patientenverfügungen in stationären Einrichtungen der Seniorenpflege: Vorkommen, Validität, Aussagekraft und Beachtung durch das Pflegepersonal. Dtsch Arztebl Int 109(37): 577-583

Trzeczak S (2013) Notfallmedizin: Ethische Kompetenz und praktische Erfahrung. Dtsch Arztebl Int 110(15): 706-707

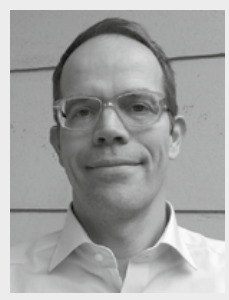

\section{Dr. med. Jan Padberg}

Nach dem Studium der Medizin in Würzburg und Berlin Ausbildung zum Facharzt für Innere Medizin an der Charité. 2002 Anerkennung Facharzt Innere Medizin, im Anschluss dreijährige Ausbildung fakultative Weiterbildung Internistische Intensivmedizin. 2004 Zusatzbezeichnung Infektiologie. Seit 2005 aktiver Notarzt in Berlin und Brandenburg. 2007 Anerkennung Facharzt Pneumologie. 2007 bis 2010 Leitender Oberarzt Intensivstation und Rettungsstelle im Gemeinschaftskrankenhaus Havelhöhe Berlin, Mitglied des Ethikkomitees. 2010-2012 Ärztlicher Leiter ZNA am Ev. Waldkrankenhaus Spandau. 2011 Zusatzbezeichnung Ärztliches Qualitätsmanagement. Seit 2012 Oberarzt des Notfallzentrums im HELIOS Klinikum Emil von Behring Berlin. 2013 Zusatzbezeichnung Notfallmedizin. Gründungsmitglied der Arbeitsgemeinschaft Ethik in der Notfallmedizin der Deutschen Gesellschaft für interdisziplinäre Notfall- und Akutmedizin (DGINA). 